demonstrated associations between $\mathrm{PA}$ and age $(\beta=-2.37$, $\mathrm{p}=0.01)$ and lean mass $(\beta=2.45, \mathrm{p}=0.002)$. PA was lower in males $(\beta=-49.84, p=0.001)$, on weekends $(\beta=-5.49, p=0.01)$ and in those who died within 1-year $(\beta=-41.24, p=0.04)$, and was associated with total sleep time (TST) $(\beta=0.01, p=0.003)$, EXACT score $(\beta=-0.97, p=0.002)$, COPD assessment test $(\beta=-$ $1.63, \mathrm{p}=0.02), \mathrm{FEV}_{1}(\beta=46.38, \mathrm{p}<0.001)$, inspiratory capacity $(\beta=44.17, \mathrm{p}<0.001), \mathrm{PI}_{\max }(\beta=2.14, \mathrm{p}<0.001)$ and neural respiratory drive, measured using parasternal EMG $(\beta=-2.12$, $\mathrm{p}=0.01)$. Patients readmitted within 28-days exhibited poorer sleep quality than non-readmitted patients (TST: $\beta=-110$, $p=0.004$, latency: $\beta=34, p=0.03$ ).

Conclusions This study provides a novel insight into the improvement in daytime activity occurring in the 28 days following hospital discharge after severe COPD exacerbation. Physical activity related inversely to age, symptom burden, health status and neural respiratory drive, and positively to lean mass, respiratory muscle strength, expiratory airflow and inspiratory capacity. Total sleep time fell following hospital discharge, and sleep quality was lower in readmitted patients. Future research is needed to evaluate the impact of targeted interventions that enhance physical activity and sleep quality on hospital readmission in this high-risk population.

\section{S30 PREDICTING HOSPITAL LENGTH OF STAY FOR ACUTE ADMISSIONS IN PATIENTS WITH COPD}

${ }^{1} \mathrm{G}$ Cox, ${ }^{1} \mathrm{~S}$ Burns, ${ }^{2} \mathrm{~A}$ Taylor, ${ }^{1} \mathrm{P}$ McGinness, ${ }^{2} \mathrm{DJ}$ Lowe, ${ }^{2} \mathrm{C}$ Carlin. ${ }^{2}$ StormID, Edinburgh, UK; ${ }^{2}$ Queen Elizabeth University Hospital, Glasgow, UK

10.1136/thorax-2021-BTSabstracts.36

Introduction Accurate predictions of hospital length of stay (LOS) at the time of admission allows clinicians to direct patients to the most appropriate medical services, prevent overcrowding in emergency departments via improved patient flow, and better manage hospital resources.

Objectives To develop, evaluate and explain machine learning classifiers that predict prolonged LOS ( $\geq 2$ days) using information that is known at the time of acute admission, does not change during the patient's hospital stay, and would be easy to input to a model deployed in a clinical setting.

Methods A SafeHaven dataset of de-identified electronic health records for acute admissions of patients with COPD to four Scottish hospitals between January 2010 and March 2019 was prepared. Using XGBoost algorithms and a binary classifier (admission $<48$ hours or $>48$ hours) we developed a set of machine-learning models that predict whether a patient will have a prolonged LOS and investigated which variables contribute the most to prediction performance. We produced separate models for: 1) all acute admissions in the study period $(\mathrm{n}=75387)$; 2) COPD related admissions $(\mathrm{n}=12137) ; 3)$ admissions relating to COPD or a broader set of respiratory conditions $(n=20134)$. We evaluated model performance on an unseen test data set based on Receiver Operating Characteristic and Precision Recall Curves, and the precision, recall and F1 scores. Further, we compared models to two established clinical scores to predict emergency department disposition: the Glasgow Admission Prediction Score (GAPS) and the Ambulatory Score (Ambs). We used SHapley Additive exPlanations to explain why specific model predictions are made for individual patients.
Results Our models highlighted several key factors that contribute to prolonged LOS in COPD patients. Some relate to patient clinical history, such as certain existing comorbidities, previous diagnoses on discharge and LOS for previous hospital visits, which is rarely considered in LOS prediction models.

Conclusions We have identified several factors relating to clinical and admission history that influence COPD patients' likelihood of prolonged acute admissions and are able to explain the rationale behind individual predictions. Since these factors would be known at admission time, they could be passed to a deployed LOS predictive model to aid clinical decision making.

\section{S31 HOME OXYGEN THERAPY AND SMOKING: PLAYING WITH FIRE?}

${ }^{1}$ TS FitzMaurice, ${ }^{2} \mathrm{C}$ Jager, ${ }^{2} \mathrm{M}$ Simmons, ${ }^{1} \mathrm{D}$ Barber, ${ }^{1} \mathrm{D}$ Wat. 'Liverpool Heart and Chest Hospital NHS Foundation Trust, Liverpool, UK; ${ }^{2}$ University of Liverpool, Liverpool, UK

\subsection{6/thorax-2021-BTSabstracts.37}

Introduction Long term oxygen therapy (LTOT) use has increased significantly in the last 40 years in chronic hypoxaemic COPD patients, as it has been shown to confer a survival benefit. Smoking is cited as a contraindication for LTOT due to elevated risks of fire. ${ }^{1}$ Despite warnings about potential dangers, some individuals continue to smoke whilst on LTOT. Methods We conducted a retrospective review of all consecutive hypoxaemic COPD patients considered for LTOT between January 2016 and December 2017, with follow up until January 2021 , in order to evaluate the survival difference between ex-smokers and current smokers with hypoxaemic COPD on LTOT, and current smokers eligible for - but not currently on - LTOT. Note was made of smoking status at time of death/ censoring. Prior to LTOT, all patients were provided with standard fire safety information and risk-reducing interventions.

Results There were 74 eligible patients (mean \pm SD average $\mathrm{ppFEV}_{1} 43 \pm 20$, BMI $28 \pm 9 \mathrm{~kg} / \mathrm{m}^{2}, 48$ female), of whom 28 were current smokers at their time of death and 46 ex-smokers. Of the 74, 13 current smokers did not receive LTOT due to fire safety concerns or individual refusal. Current smokers had a significantly lower BMI $(26 \pm 11$ vs $49 \pm 21, \mathrm{P}<0.05)$ and $\operatorname{ppFEV}_{1}\left(25 \pm 10\right.$ vs $\left.28 \pm 8 \mathrm{~kg} / \mathrm{m}^{2}, \mathrm{P}<0.05\right)$ than ex-smokers. Comorbidities and age were similar between groups. Survival was assessed using a Kaplan-Meier plot. Survival at 5 years did not differ significantly between current smokers and ex-smokers on LTOT $(\mathrm{P}>0.9)$, but survival between current smokers on LTOT versus those not on LTOT was significant $(\mathrm{P}<0.001)$. No fire incidents were recorded during the study period.

Conclusions Smoking on LTOT is controversial, with arguments made for discrimination and the conflict between the right to smoke, versus the risk of harm to self and others. Whilst every effort should be made to discourage smoking in any form, LTOT may confer survival benefits to carefully riskassessed smokers under close supervision. A larger study may provide more accurate survival data of LTOT in this cohort.

\section{REFERENCE}

1. National Institute for Health and Care Excellence. Chronic obstructive pulmonary disease in over 16s: diagnosis and management, 2018. 